International Journal of Pure and Applied Mathematics

Volume 100 No. 4 2015, 497-506

ISSN: 1311-8080 (printed version); ISSN: 1314-3395 (on-line version)

url: http://www.ijpam.eu

doi: http://dx.doi.org/10.12732/ijpam.v100i4.9

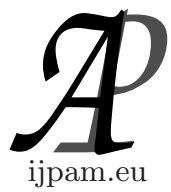

\title{
OSCULATING CURVES IN THE GALILEAN 4-SPACE
}

\author{
Dae Won Yoon ${ }^{1}$, Jae Won Lee ${ }^{2}$, Chul Woo Lee ${ }^{3 \S}$ \\ ${ }^{1}$ Department of Mathematics Education and RINS \\ Gyeongsang National University \\ Jinju, 660-701, REPUBLIC OF KOREA \\ ${ }^{2}$ Department of Mathematics Education \\ Busan National University of Education \\ Busan 611-736, REPUBLIC OF KOREA \\ ${ }^{3}$ Department of Mathematics \\ Kyungpook National University \\ Daegu, 702-701, REPUBLIC OF KOREA
}

\begin{abstract}
In this paper, we study osculating curves and equiform osculating curves in the 4-dimensional Galilean space $G_{4}$ and characterize such curves in terms of their curvature functions and their equiform curvature functions, respectively.
\end{abstract}

AMS Subject Classification: 53A35, 53C44

Key Words: Galilean space, equiform geometry, osculating curve

\section{Introduction}

In the Euclidean space $\mathbb{E}^{3}$, there exist three classes of curves, called rectifying, normal, and osculating curves, which satisfy the Cesaro's fixed point condition (see [5]). Namely, rectifying, normal, and osculating planes of such curves always contain a particular point. It is well-known that if all the normal or osculating planes of a curve in $\mathbb{E}^{3}$ pass through a particular point, then the

Received: January 5, 2015

(C) 2015 Academic Publications, Ltd.

${ }^{\S}$ Correspondence author url: www.acadpubl.eu 
curve lies in a sphere or is a planner curve, respectively. It is also known that if all rectifying planes of a non-planar curve in $\mathbb{E}^{3}$ pass through a particular point, then the ratio of torsion and curvature of such curve is a non-constant linear function (see [2]). Moreover, İlarslan and Nešovic (see [4]) gave some characterizations for osculating curves in $\mathbb{E}^{3}$, and they also constructed osculating curves in $\mathbb{E}^{4}$ as a curve whose position vector all the time lies in the orthogonal complement of its first binormal vector field. As the results, they classified the osculating curves in terms of their curvature functions and gave the necessary and the sufficient conditions of osculating curves for arbitrary curves in $\mathbb{E}^{4}$.

On the other hand, the equiform geometry of the Cayley-Klein space is defined by requesting that similarity group of the space preserves angles between planes and lines. Cayley-Klein geometries are studied for many years. However, they recently have become interesting again because of their importance for other fields, like soliton theory (see [7])

A Galilean space is one of the Cayley-Klein spaces and it has been largely developed by Röschel (see [6]). A Galilean space may be considered as the limit case of a pseudo-Euclidean space in which the isotropic cone degenerates to a plane. The limit transition corresponds to the limit transition from the special relatively theory to classical mechanics.

In this paper, we study osculating curves and equiform osculating curves in the 4-dimensional Galilean space $G_{4}$ and characterize such curves in terms of their curvature functions and their equiform curvature functions, respectively.

\section{Preliminaries}

The 3-dimensional Galilean space $G_{3}$ is the Cayley-Klein space equipped with the projective metric of signature $(0,0,+,+)$. The absolute figure of the Galilean space consists of an ordered triple $\{w, f, I\}$, where $w$ is the ideal (absolute) plane, $f$ is the line (absolute line) in $w$ and $I$ is the fixed elliptic involution of points of $f$.

The study of mechanics of plane-parallel motions reduces to the study of a geometry of the 3-dimensional space with coordinates $\{x, y, t\}$, given by the motion formula. It is explained that the 4-dimensional Galilean geometry, which studies all properties invariant under motions of objects in the space, is even complex.

In addition, it is started that this geometry can be described more precisely as the study of those properties of the 4-dimensional space with coordinates 
which are invariant under the general Galilean transformations as follows [8]:

$$
\begin{aligned}
x^{\prime}= & (\cos \beta \cos \alpha-\cos \gamma \sin \beta \sin \alpha) x+(\sin \beta \cos \alpha \\
& -\cos \gamma \cos \beta \sin \alpha) y+(\sin \gamma \sin \alpha) z+\left(v \cos \delta_{1}\right) t+a, \\
y^{\prime}= & -(\cos \beta \sin \alpha+\cos \gamma \sin \beta \cos \alpha) x+(-\sin \beta \sin \alpha \\
& -\cos \gamma \cos \beta \cos \alpha) y+(\sin \gamma \cos \alpha) z+\left(v \cos \delta_{2}\right) t+b, \\
z^{\prime}= & (\sin \gamma \sin \beta) x-(\sin \gamma \cos \beta) y+(\cos \gamma) z+\left(v \cos \delta_{3}\right) t+c, \\
t^{\prime}= & t+d,
\end{aligned}
$$

where $\cos ^{2} \delta_{1}+\cos ^{2} \delta_{2}+\cos ^{2} \delta_{3}=1$.

The Galilean scalar product in $G_{4}$ can be written as

$$
\langle\mathbf{x}, \mathbf{y}\rangle= \begin{cases}x_{1} y_{1}, & \text { if } \quad x_{1} \neq 0 \quad \text { or } \quad y_{1} \neq 0 \\ x_{2} y_{2}+x_{3} y_{3}+x_{4} y_{4}, & \text { if } \quad x_{1}=0 \quad \text { and } \quad y_{1}=0\end{cases}
$$

where $\mathbf{x}=\left(x_{1}, x_{2}, x_{3}, x_{4}\right)$ and $\mathbf{y}=\left(y_{1}, y_{2}, y_{3}, y_{4}\right)$ are vectors in $G_{4}$. It leaves invariant the Galilean norm of the vector $\mathbf{x}$, defined by

$$
\|\mathbf{x}\|=\left\{\begin{array}{lll}
\left|x_{1}\right|, & \text { if } & x_{1} \neq 0 \\
\sqrt{x_{2}^{2}+x_{3}^{2}+x_{4}^{2}}, & \text { if } & x_{1}=0 .
\end{array}\right.
$$

The Galilean cross product of $\mathbf{x}, \mathbf{y}$ and $\mathbf{z}$ on $G_{4}$ is defined by

$$
\mathbf{x} \times \mathbf{y} \times \mathbf{z}=\left|\begin{array}{cccc}
0 & e_{2} & e_{3} & e_{4} \\
x_{1} & x_{2} & x_{3} & x_{4} \\
y_{1} & y_{2} & y_{3} & y_{4} \\
z_{1} & z_{2} & z_{3} & z_{4}
\end{array}\right|
$$

where $e_{2}=(0,1,0,0), e_{3}=(0,0,1,0)$, and $e_{4}=(0,0,0,1)$.

A curve $\alpha: I \subset \mathbb{R} \rightarrow G_{4}$ of the class $C^{\infty}$ in the Galilean space $G_{4}$ is defined by the parametrization

$$
\alpha(s)=(s, y(s), z(s), w(s)),
$$

where $s$ is a Galilean invariant arc-length of $\alpha$. 
On the other hand, the Frenet vectors of $\alpha(s)$ in $G_{4}$ are defined by

$$
\begin{aligned}
\mathbf{t}(s) & =\frac{d \alpha(s)}{d s}=\dot{\alpha}(s)=(1, \dot{y}(s), \dot{z}(s), \dot{w}(s)), \\
\mathbf{n}(s) & =\frac{1}{\kappa_{1}(s)} \ddot{\alpha}(s)=\frac{1}{\kappa_{1}(s)}(0, \ddot{y}(s), \ddot{z}(s), \ddot{w}(s)), \\
\mathbf{b}_{1}(s) & =\frac{1}{\kappa_{2}(s)}\left(0, \frac{d\left(\frac{1}{\kappa_{1}(s)} y(s)\right)}{d s}, \frac{d\left(\frac{1}{\kappa_{1}(s)} z(s)\right)}{d s}, \frac{d\left(\frac{1}{\kappa_{1}(s)} w(s)\right)}{d s}\right), \\
\mathbf{b}_{2}(s) & =\mathbf{t}(s) \times \mathbf{n}(s) \times \mathbf{b}_{1}(s),
\end{aligned}
$$

where $\kappa_{1}(s), \kappa_{2}(s)$, and $\kappa_{3}(s)$ are the first, second and third curvature functions, respectively, given by

$$
\begin{aligned}
& \kappa_{1}(s)=\sqrt{\ddot{y}(s)^{2}+\ddot{z}(s)^{2}+\ddot{w}(s)^{2}}, \\
& \kappa_{2}(s)=\sqrt{\langle\dot{n}(s), \dot{n}(s)\rangle}, \\
& \kappa_{3}(s)=\left\langle\dot{b}_{1}(s), b_{2}(s)\right\rangle .
\end{aligned}
$$

The vectors $\mathbf{t}, \mathbf{n}, \mathbf{b}_{1}, \mathbf{b}_{2}$ are called the tangent, principal normal, first binormal, and second binormal vectors of $\alpha$, respectively. If the curvature functions $\kappa_{1}, \kappa_{2}$ and $\kappa_{3}$ of $\alpha$ are constants, then a curve $\alpha$ is called a $W$-curve. For their derivatives the following Frenet formula satisfies (cf. [3])

$$
\begin{aligned}
\dot{\mathbf{t}}(s) & =\kappa_{1}(s) \mathbf{n}(s), \\
\dot{\mathbf{n}}(s) & =\kappa_{2}(s) \mathbf{b}_{1}(s), \\
\dot{\mathbf{b}_{1}}(s) & =-\kappa_{2}(s) \mathbf{n}(s)+\kappa_{3}(s) \mathbf{b}_{2}(s), \\
\dot{\mathbf{b}_{2}}(s) & =-\kappa_{3}(s) \mathbf{b}_{1}(s) .
\end{aligned}
$$

Now, we define osculating curves in the Galilean space $G_{4}$.

Let $\alpha$ be a unit speed curve in $G_{4}$. If its position vector always lies in the orthogonal complement $\mathbf{b}_{1}^{\perp}$ or $\mathbf{b}_{2}^{\perp}$ of $\mathbf{b}_{1}$ or $\mathbf{b}_{2}$, then a curve $\alpha$ is called an osculating curve in $G_{4}$. Consequently, an osculating curve can be expressed as

$$
\alpha(s)=\lambda(s) \mathbf{t}(s)+\mu(s) \mathbf{n}(s)+\nu(s) \mathbf{b}_{2}(s),
$$

or

$$
\alpha(s)=\lambda(s) \mathbf{t}(s)+\mu(s) \mathbf{n}(s)+\nu(s) \mathbf{b}_{1}(s)
$$

for some smooth functions $\lambda(s), \mu(s)$ and $\nu(s)$.

In this paper, we deal with an osculating curve generating by the tangent vector, the principal normal vector and the second binormal vector of the curve $\alpha$ in $G_{4}$. 


\section{Osculating Curves in $G_{4}$}

In this section, we characterize osculating curves in $G_{4}$ in terms of their curvatures.

Theorem 1. Let $\alpha$ be a unit speed curve in $G_{4}$ with non-zero curvatures $\kappa_{1}, \kappa_{2}$ and $\kappa_{3}$. Then $\alpha$ is an osculating curve if and only if

$$
-\frac{d}{\kappa_{1}}\left(\frac{\dot{\kappa_{3}}}{\kappa_{2}}\right)=s+c
$$

where $c, d \neq 0$ are constant.

Proof. Let $\alpha=\alpha(s)$ be a unit speed osculating curve and $\kappa_{1}(s), \kappa_{2}(s)$, and $\kappa_{3}(s)$ be non-zero curvatures of $\alpha$. Then, the position vector $\alpha(s)$ of the curve $\alpha$ satisfies the following equation:

$$
\alpha(s)=\lambda(s) \mathbf{t}(s)+\mu(s) \mathbf{n}(s)+\nu(s) \mathbf{b}_{2}(s)
$$

for some smooth functions $\lambda(s), \mu(s)$ and $\nu(s)$. Differentiating the above equation with respect to $s$ with the Frenet formulae (2.2), we obtain

$$
\mathbf{t}=\dot{\lambda} \mathbf{t}+\left(\kappa_{1} \lambda+\dot{\mu}\right) \mathbf{n}+\left(\kappa_{2} \mu-\nu \kappa_{3}\right) \mathbf{b}_{1}+\dot{\nu} \mathbf{b}_{2} .
$$

From this, we get

$$
\begin{cases}\dot{\lambda} & =1 \\ \kappa_{1} \lambda+\dot{\mu} & =0 \\ \kappa_{2} \mu-\nu \kappa_{3} & =0 \\ \dot{\nu} & =0\end{cases}
$$

It follows that

$$
\left\{\begin{array}{l}
\lambda=s+c \\
\mu=\frac{\kappa_{3}}{\kappa_{2}} d \\
\nu=d, \quad c, d \neq 0 \in \mathbb{R} .
\end{array}\right.
$$

Using the second equation in (3.2) and (3.3), we obtain the curvatures $\kappa_{1}, \kappa_{2}$ and $\kappa_{3}$ satisfying the equation

$$
-\frac{d}{\kappa_{1}}\left(\frac{\dot{\kappa_{3}}}{\kappa_{2}}\right)=s+c .
$$


Conversely, assume that the curvatures $\kappa_{1}(s), \kappa_{2}(s)$ and $\kappa_{3}(s)$ of a unit speed curve $\alpha$ in $G_{4}$ satisfy equation (3.4). Let us consider the vector $\mathbf{x} \in G_{4}$, given by

$$
\mathbf{x}(s)=\alpha(s)-(s+c) \mathbf{t}(s)-\frac{d \kappa_{3}}{\kappa_{2}} \mathbf{n}(s)-d \mathbf{b}_{2} .
$$

Then, we can easily find $\dot{\mathbf{x}}(s)=0$, that is, $\mathbf{x}$ is a constant vector. Thus, $\alpha$ is an osculating curve.

From (3.1), we have the following:

Theorem 2. None of a unit speed $W$-curve with non-zero curvatures $\kappa_{1}$, $\kappa_{2}$ and $\kappa_{3}$ in $G_{4}$ is an osculating curve.

Remark 3. The above theorem gives the opposite result from the case of the Euclidean space (see [4]).

Theorem 4. Let $\alpha$ be a unit speed osculating curve in $G_{4}$ with non-zero curvatures $\kappa_{1}, \kappa_{2}$ and $\kappa_{3}$. Then the following statements hold:

$$
\begin{cases}\frac{\kappa_{3}}{\kappa_{2}} & =\int\left(-\frac{\kappa_{1}}{d}(s+c)\right) d s, \\ \langle\alpha(s), \mathbf{t}(s)\rangle & =s+c, \\ \langle\alpha(s), \mathbf{n}(s)\rangle & =\frac{\kappa_{3}}{\kappa_{2}} d, \\ \left\langle\alpha(s), \mathbf{b}_{2}(s)\right\rangle & =d, d \in \mathbb{R}-\{0\} .\end{cases}
$$

Conversely, if $\alpha$ is a unit speed curve in $G_{4}$ with non-zero curvatures $\kappa_{1}, \kappa_{2}$ and $\kappa_{3}$ with one of equations in (3.5), then $\alpha$ is an osculating curve .

Proof. It is clear from equations (3.1), (3.2) and (3.3).

From Theorem 4, we have

Theorem 5. A unit speed osculating curve in $G_{4}$ with non-zero curvatures $\kappa_{1}, \kappa_{2}$ and $\kappa_{3}$ is given by

$$
\alpha(s)=(s+c) \mathbf{t}(s)-\left(\int \kappa_{1}(s-c) d s\right) \mathbf{n}(s)+d \mathbf{b}_{2}(s) .
$$




\section{Frenet Formulas in Equiform Geometry in $G_{4}$}

Let $\alpha: I \rightarrow G_{4}$ be a curve in the Galilean space $G_{4}$. We define the equiform parameter of $\alpha$ by

$$
\sigma:=\int \frac{1}{\rho} d s=\int \kappa d s,
$$

where $\rho=\frac{1}{\kappa}$ is the radius of curvature of the curve $\alpha$. Then, we have

$$
\frac{d s}{d \sigma}=\rho .
$$

Let $h$ be a homothety with the center in the origin and the coefficient $\lambda$. If we put $\tilde{\alpha}=h(\alpha)$, then it follows

$$
\tilde{s}=\lambda s \quad \text { and } \quad \tilde{\rho}=\lambda \rho,
$$

where $\tilde{s}$ is the arc-length parameter of $\tilde{\alpha}$ and $\tilde{\rho}$ the radius of curvature of this curve. Therefore, $\sigma$ is an equiform invariant parameter of $\alpha$ (see [4]).

From now on, we define the Frenet formula of the curve $\alpha$ with respect to the equiform invariant parameter $\sigma$ in $G_{4}$.

The vector

$$
\mathrm{T}=\frac{d \alpha}{d \sigma}
$$

is called a tangent vector of the curve $\alpha$. From (2.1) and (2.2), we get

$$
\mathrm{T}=\frac{d \alpha}{d s} \cdot \frac{d s}{d \sigma}=\rho \cdot \frac{d \alpha}{d s}=\rho \cdot \mathbf{t} .
$$

We define the principal normal vector, the first binormal vector and the second binormal vector by

$$
\mathrm{N}=\rho \cdot \mathbf{n}, \quad \mathrm{B}_{1}=\rho \cdot \mathbf{b}_{1} \quad \mathrm{~B}_{2}=\rho \cdot \mathbf{b}_{2} .
$$

Then, we easily show that $\left\{\mathrm{T}, \mathrm{N}, \mathrm{B}_{1}, \mathrm{~B}_{2}\right\}$ is an equiform invariant tetrahedron of the curve $\alpha$.

On the other hand, the derivations of these vectors with respect to $\sigma$ are given by

$$
\begin{aligned}
\mathrm{T}^{\prime} & =\frac{d \mathrm{~T}}{d \sigma}=\dot{\rho} \mathrm{T}+\mathrm{N} \\
\mathrm{N}^{\prime} & =\frac{d \mathrm{~N}}{d \sigma}=\dot{\rho} \mathrm{N}+\frac{\kappa_{2}}{\kappa_{1}} \mathrm{~B}_{1}, \\
\mathrm{~B}_{1}^{\prime} & =\frac{d \mathrm{~B}_{1}}{d \sigma}=-\frac{\kappa_{2}}{\kappa_{1}} \mathrm{~N}+\dot{\rho} \mathrm{B}_{1}+\frac{\kappa_{3}}{\kappa_{1}} \mathrm{~B}_{2} \\
\mathrm{~B}_{2}^{\prime} & =\frac{d \mathrm{~B}_{2}}{d \sigma}=-\frac{\kappa_{3}}{\kappa_{1}} \mathrm{~B}_{1}+\dot{\rho} \mathrm{B}_{2} .
\end{aligned}
$$


Definition. The function $\mathcal{K}_{i}: I \rightarrow \mathbb{R}(i=1,2,3)$, defined by

$$
\mathcal{K}_{1}=\dot{\rho}, \mathcal{K}_{2}=\frac{\kappa_{2}}{\kappa_{1}}, \mathcal{K}_{3}=\frac{\kappa_{3}}{\kappa_{1}}
$$

is called the $i^{\text {th }}$ equiform curvature of the curve $\alpha$.

Thus, the formula analogous to the Frenet formula in the equiform geometry of the Galilean space have the following form [1]

$$
\begin{aligned}
\mathrm{T}^{\prime} & =\mathcal{K}_{1} \cdot \mathrm{T}+\mathrm{N} \\
\mathrm{N}^{\prime} & =\mathcal{K}_{1} \cdot \mathrm{N}+\mathcal{K}_{2} \cdot \mathrm{B}_{1} \\
\mathrm{~B}_{1}^{\prime} & =-\mathcal{K}_{2} \cdot \mathrm{N}+\mathcal{K}_{1} \cdot \mathrm{B}_{1}+\mathcal{K}_{3} \cdot \mathrm{B}_{2} \\
\mathrm{~B}_{2}^{\prime} & =-\mathcal{K}_{3} \cdot \mathrm{B}_{1}+\mathcal{K}_{1} \cdot \mathrm{B}_{2} .
\end{aligned}
$$

\section{Osculating Curves According to Equiform in $G_{4}$}

Let $\alpha$ be osculating curve in $G_{4}$ with non-zero equiform curvatures $\mathcal{K}_{1}, \mathcal{K}_{2}$, and $\mathcal{K}_{3}$ with respect to the equiform invariant parameter $\sigma$. Then, the radius vector $\alpha(\sigma)$ of the curve $\alpha$ secures the following equation:

$$
\alpha=\lambda \mathrm{T}+\mu \mathrm{N}+\nu \mathrm{B}_{2}
$$

for some differentiable functions $\lambda, \mu$ and $\nu$ in $G_{4}$. Therefore, we put up the following theorems of osculating curve $\alpha$. Differentiating the osculating curve with respect to $\sigma$ and using the equations (4.4), we obtain

$$
T=\left(\lambda^{\prime}+\lambda \mathcal{K}_{1}\right) T+\left(\lambda+\mu^{\prime}+\mu \mathcal{K}_{1}\right) N+\left(\mu \mathcal{K}_{2}-\nu \mathcal{K}_{3}\right) B_{1}+\left(\nu^{\prime}+\nu \mathcal{K}_{1}\right) B_{2}
$$

It follows that

$$
\begin{cases}\lambda^{\prime}+\lambda \mathcal{K}_{1} & =1 \\ \lambda+\mu^{\prime}+\mu \mathcal{K}_{1} & =0 \\ \mu \mathcal{K}_{2}-\nu \mathcal{K}_{3} & =0 \\ \nu^{\prime}+\nu \mathcal{K}_{1} & =0\end{cases}
$$

and therefore,

$$
\left\{\begin{array}{l}
\lambda=\left(\frac{c \mathcal{K}_{3}}{\mathcal{K}_{2}}\right)^{\prime} e^{-\int \mathcal{K}_{1} d \sigma} \\
\mu=\frac{c \mathcal{K}_{3} e^{-\int \mathcal{K}_{1} \mathrm{d \sigma}}}{\mathcal{K}_{2}} \\
\nu=c e^{-\int \mathcal{K}_{1} d \sigma}
\end{array}\right.
$$


where $c$ is non-zero constant. In this way, the functions $\lambda(\sigma), \mu(\sigma)$, and $\nu(\sigma)$ are expressed in terms of the equiform curvatures $\mathcal{K}_{1}, \mathcal{K}_{2}$, and $\mathcal{K}_{3}$ of the curve $\alpha$. Moreover, by using the first equation in (5.1) and relation (5.2), we easily find that equiform curvature functions $\mathcal{K}_{1}, \mathcal{K}_{2}$, and $\mathcal{K}_{3}$ satisfy the equation

$$
\left(\frac{\mathcal{K}_{3}}{\mathcal{K}_{2}}\right)^{\prime \prime}=\frac{1}{c} e^{\int \mathcal{K}_{1} d \sigma}
$$

Conversely, assume that the equiform curvatures $\mathcal{K}_{1}, \mathcal{K}_{2}$, and $\mathcal{K}_{3}$ of an arbitrary unit speed curve $\alpha$ in $G_{4}$ satisfy the euqation (5.3). Let us consider the vector $X \in G_{4}$ given by

$$
X(\sigma)=\alpha(\sigma)-\left(\frac{c \mathcal{K}_{3}}{\mathcal{K}_{2}}\right)^{\prime} e^{-\int \mathcal{K}_{1} d \sigma} T(\sigma)-\frac{c \mathcal{K}_{3} e^{-\int \mathcal{K}_{1} d \sigma}}{\mathcal{K}_{2}} N(\sigma)-c e^{-\int \mathcal{K}_{1} d \sigma} B_{2}(\sigma) .
$$

From the relations (4.4) and (5.3), we have $X^{\prime}(\sigma)=0$, which means that $X$ is a constant vector. This implies that $\alpha$ is congruent to an osculating curve. In this process, we have the following theorem.

Theorem 6. Let $\alpha(\sigma)$ be a unit speed curve in equiform geometry in $G_{4}$ with non-zero equiform curvatures $\mathcal{K}_{1}, \mathcal{K}_{2}$, and $\mathcal{K}_{3}$. Then $\alpha(\sigma)$ is congruent to an osculating curve if and only if

$$
\left(\frac{\mathcal{K}_{3}}{\mathcal{K}_{2}}\right)^{\prime \prime}=\frac{1}{c} e^{\int \mathcal{K}_{1} d \sigma}, \quad c \in \mathbb{R}
$$

From (5.3), we obtain the following theorem.

Theorem 7. There are no osculating curves lying fully in $G_{4}$ with nonzero constant equiform curvatures $\mathcal{K}_{2}$ and $\mathcal{K}_{3}$.

Theorem 8. Let $\alpha(\sigma)$ be unit speed curve in equiform geometry of $G_{4}$ with non-zero equiform curvatures $\mathcal{K}_{1}, \mathcal{K}_{2}$, and $\mathcal{K}_{3}$. Then $\alpha(\sigma)$ is congruent to an osculating curve if $\mathcal{K}_{1}=$ constant $\neq 0$ and $\frac{\mathcal{K}_{3}}{\mathcal{K}_{2}}(\sigma)=\frac{1}{c \mathcal{K}_{1}^{2}} e^{\mathcal{K}_{1} \sigma}+d_{1} \sigma+d_{2}, \quad c, d_{1}, d_{2} \in \mathbb{R}$.

Proof. Suppose that $\mathcal{K}_{1}=$ constant $\neq 0$. By using the equation (5.3), we find differential equation

$$
\left(\frac{\mathcal{K}_{3}}{\mathcal{K}_{2}}\right)^{\prime \prime}=\frac{1}{c} e^{\mathcal{K}_{1} \sigma}
$$


The solution of the previous differential equation is given by

$$
\frac{\mathcal{K}_{3}}{\mathcal{K}_{2}}(\sigma)=\frac{1}{c \mathcal{K}_{1}^{2}} e^{\mathcal{K}_{1} \sigma}+d_{1} \sigma+d_{2}, \quad c, d_{1}, d_{2} \in \mathbb{R} .
$$

\section{Acknowledgments}

The first author was supported by Basic Science Research Program through the National Research Foundation of Korea (NRF) funded by the Ministry of Education, Science and Technology (2012R1A1A2003994).

\section{References}

[1] M.E. Aydin, M. Ergüt, The equiform differential geometry of curves in 4dimensional galilean space $G_{4}$, Stud. Univ. Babeş-Bolyai Math., 58 (2013), 399-406.

[2] B.Y. Chen, When does the position vector of a space curve always lie in its rectifying plane?, Amer. Math. Monthly, 110 (2003), 147-152.

[3] Z. Erjavec, B. Divjak, The equiform differential geometry of curves in the pseudo-Galilean space, Math. Communications, 13 (2008), 321-332.

[4] K. İlarslan, E. Nešović, Some characterizations of osculating curves in the Euclidean spaces, Demonstration Math., XLI (2008), 931-939.

[5] T. Otsuki, Differential Geometry, Asakura Pulishing Co. Ltd., Tokyo, 1961.

[6] O. Roschel, Die Geometrie Des Galileischen Raumes, Berichte der Math.Stat. Sektionim Forschumgszentrum Graz, Ber., 256 (1986), 1-20.

[7] C. Rogers, W.K. Schief, Backlund and Darboux Transformations, Geometry and Modern Applications in Soliton Theory, Cambridge University Press, 2002.

[8] I. Yaglom, A Simple Non-Euclidean Geometry and its Physical Basis, Spriger-Verlag, New York Inc., 1979. 